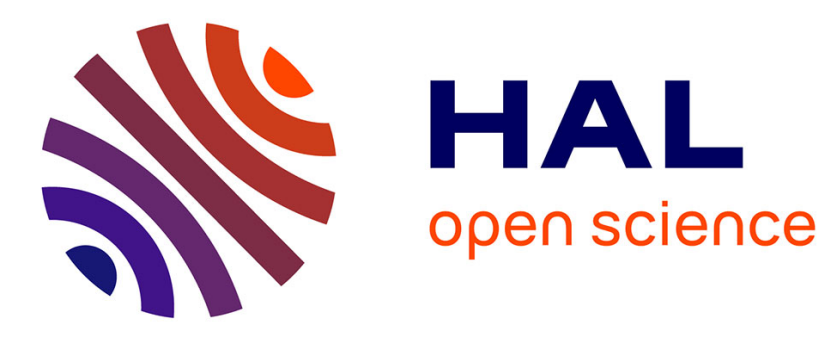

\title{
A Wearable Sensor Network for Gait Analysis: A 6-Day Experiment of Running Through the Desert
}

Guillaume Chelius, Christophe Braillon, Maud Pasquier, Nicolas Horvais, Roger Pissard-Gibollet, Bernard Espiau, Christine Azevedo Coste

\section{- To cite this version:}

Guillaume Chelius, Christophe Braillon, Maud Pasquier, Nicolas Horvais, Roger Pissard-Gibollet, et al.. A Wearable Sensor Network for Gait Analysis: A 6-Day Experiment of Running Through the Desert. IEEE/ASME Transactions on Mechatronics, 2011, 16 (5), pp.878-883. 10.1109/TMECH.2011.2161324 . lirmm-00604988

\section{HAL Id: lirmm-00604988 \\ https://hal-lirmm.ccsd.cnrs.fr/lirmm-00604988}

Submitted on 7 Mar 2014

HAL is a multi-disciplinary open access archive for the deposit and dissemination of scientific research documents, whether they are published or not. The documents may come from teaching and research institutions in France or abroad, or from public or private research centers.
L'archive ouverte pluridisciplinaire HAL, est destinée au dépôt et à la diffusion de documents scientifiques de niveau recherche, publiés ou non, émanant des établissements d'enseignement et de recherche français ou étrangers, des laboratoires publics ou privés. 
The aforementioned statistical models for estimating the fatigue levels are obtained by analyzing the data of [1]. The variables $z_{i}(i=\{1 \ldots$ $6\})$ and $y_{j}(j=\{1 \ldots 5\})$ are representative of the various cues and the fatigue values. For further information on these variables, the reader is directed to the original paper [1].

\section{B. ANN-Based Experts}

The data from [1] was used as training set for training five neural networks (one for each mode of cues). The MATLAB source codes for the networks were downloaded from collection of open-source codes from Phil Brierley's website (http://philbierley.com) which were modified to suit the current implementation. The networks consisted of one input (two inputs in case of EEG analysis) and one output (fatigue). One layer of three hidden neurons was implemented.

\section{SBN-Based Experts}

The SBN-based experts for inferring fatigue from the various cues were constructed by using an approximate guess of the prior probabilities. There are all simple cause-effect links with individual cues as the cause and fatigue state as the effect. The guesses of the prior probabilities were made by manually modifying the prior probabilities values until an acceptable accuracy of inference was achieved (see Table I). As these values were not taken from any previous studies in the literature, it is not surprising that the SBN-based experts performed the poorest in inference.

\section{TSK-Based Experts}

The TSK-based experts were used "as reported" in [1]. The source codes were obtained from the authors of [1].

\section{ACKNOWLEDGMENT}

The authors would like to thank the graduate students at the Advanced Engineering Design Laboratory, University of Saskatchewan, for the valuable suggestions. They would also like to thank the anonymous reviewers for helpful and constructive comments.

\section{REFERENCES}

[1] G. Yang, Y. Lin, and P. Bhattacharya, "A method for multi-modality inferring of human cognitive states based on the integration of neurofuzzy network and information fusion techniques," EURASIP J. Adv. Signal Process., vol. 2008, article ID 371621, pp. 10-24, 2008.

[2] Y. Lin, "Affective driving," Emotional Engineering: Service Development, Shuichi Fukuda, Ed., New York, Springer, 2010, ch. 14.

[3] C. D. He and C. C. Zhao, "Evaluation of the critical value of driving fatigue based on the fuzzy sets theory," Environ. Res., vol. 61, pp. 150-156, 1993.

[4] G. Hamonda and F. F. Saccomanno, "Neural network model for truck driver fatigue accident detection," in Proc. IEEE Can. Conf. Electr. Comput. Eng., Montreal, QC, Canada, Sep. 1995, vol. 1, pp. 362-365.

[5] T. P. Jung, S. Makeig, M. Stensmo, and T. J. Sejnowski, "Estimating alertness from the EEG power spectrum," IEEE Trans. Biomed. Eng., vol. 44, no. 1, pp. 60-69, Jan. 1997.

[6] R. W. Picard, E. Vyzas, and J. A. Healey, "Toward machine emotional intelligence: Analysis of affective physiological state," IEEE Trans. Pattern Anal. Mach. Intell., vol. 23, no. 10, pp. 1175-1191, Oct. 2001.

[7] W. Horng, C. Chen, Y. Chang, and C. Fan, "Driver fatigue detection based on the eye tracking and dynamic template matching," in Proc. IEEE Int. Conf. Netw., Sensing Control, Taiwan, 2004, vol. 1, pp. 7-12.

[8] Q. Ji, Z. Zhu, and P. Lan, "Real-time nonintrusive monitoring and prediction of driver fatigue," IEEE Trans. Vehicular Technol., vol. 53, no. 4 pp. 1052-1068, Jul. 2004

[9] G. Yang, Y. Lin, and P. Bhattacharya, "A driver fatigue recognition model based on information fusion and dynamic Bayesian network," Inf. Sci., vol. 180, pp. 1942-1954, 2010
[10] Q. Ji, P. Lan, and C. Looney, "A probabilistic framework for modeling and real-time monitoring human fatigue," IEEE Trans. Syst. Man, Cybern.: Part A, vol. 36, no. 5, pp. 862-875, Sep. 2006.

[11] X. Li and Q. Ji, "Active affective state detection and user assistance with dynamic Bayesian networks," IEEE Trans. Syst. Man, Cybern. A, vol. 35 no. 1, pp. 93-105, Jan. 2005.

[12] C. Wu, O. Tsimhoni, and Y. Liu, "Development of an adaptive workload management system using the Queueing network-model human processor (QN-MHP)," IEEE Trans. Intell. Transp. Syst., vol. 9, no. 3, pp. 463-475, Sep. 2008.

[13] Y. Liu, R. Feyen, and O. Tsimhoni, "Queueing network-model human processor (QN-MHP): A computational architecture for multitask performance in human-machine systems," ACM Trans. Comput.-Human Interaction, vol. 13, no. 1, pp. 37-70, Mar. 2006.

[14] W. D. Gray, Integrated Models of Cognitive Systems. New York: Oxford Univ. Press, 2007.

[15] R. R. Yager, "OWA aggregation over a continuous interval argument with applications to decision making," IEEE Trans. Syst., Man, Cybern. B, vol. 34, no. 5, pp. 1952-1963, Oct. 2004.

[16] R. T. Clemen and R. L. Winkler, "Combining probability distribution from experts in risk analsysis," Risk Analysis, vol. 19, pp. 187-203, 1999.

\section{A Wearable Sensor Network for Gait Analysis: A Six-Day Experiment of Running Through the Desert}

\author{
Guillaume Chelius, Christophe Braillon, Maud Pasquier, \\ Nicolas Horvais, Roger Pissard Gibollet, Bernard Espiau, \\ and Christine Azevedo Coste
}

\begin{abstract}
This paper presents a new system for analysis of walking and running gaits. The system is based on a network of wireless nodes with various types of embedded sensors. It has been designed to allow long-term recording in outdoor environments and was tested during the 2010 "Sultan Marathon des Sables" desert race. A runner was fitted with the sensory network for six days of the competition. Although technical problems have limited the amount of data recorded, the experiment was nevertheless successful: the system did not interfere with the runner, who finished with a high ranking, the concept was validated and high quality data were acquired. It should be noted that the loss of some of the measurements was mainly due to problems with the cable connectors between the nodes and batteries. In this paper, we describe the technical aspects of the system developed, the experimental conditions under which it was validated, and give examples of the data obtained with some preliminary processing.
\end{abstract}

Index Terms-Biomechatronics, gait, sport, wireless sensors.

Manuscript received November 16, 2010; revised March 15, 2011; accepted June 1, 2011. Date of publication August 4, 2011; date of current version August 30, 2011. Recommended by Guest Editor A. Mihailidis.

G. Chelius is with the Université de Lyon, INRIA, ENS Lyon, 69342 Lyon, France (e-mail: guillaume.chelius@inria.fr).

C. Braillon, M. Pasquier, R. P. Gibollet, and B. Espiau are with the INRIA Laboratory, 38000 Grenoble, France (e-mail: christophe.braillon@ inrialpes.fr; maud.pasquier@inrialpes.fr; roger.pissard-gibollet@inrialpes.fr; bernard.espiau@inria.fr).

N. Horvais is with SALOMON Corporation, 74996 Annecy, France (e-mail: nicolas.horvais@salomon.com).

C. A. Coste is with the INRIA/LIRMM Laboratory, 34392 Montpellier, France (e-mail: azevedo@lirmm.fr).

Color versions of one or more of the figures in this paper are available online at http://ieeexplore.iee.org.

Digital Object Identifier 10.1109/TMECH.2011.2161324 


\section{INTRODUCTION}

Analysis of the motion of living organisms from various species lies at the heart of many areas of research and their applications. In fundamental research, studies in biomechanics, neurosciences, ecology, and animal biology often require recording of posture and self-propulsion data, as well as data on bulk movement of individuals or populations. Beyond these established fields of investigation, motion analysis is continually developing larger and more varied applications.

In the field of sport (see, [1] for a review), the two main areas of interest are, first, to use accurate measurements to improve an athlete's individual performance and, second, the design of "better" sportswear, shoes, or sports equipment by evaluating its efficiency using motion analysis.

The required motion data may include, for example, joint angles, position, velocity, and acceleration of limbs, ground reaction forces, and the overall motion of the body with respect to a reference frame on the ground. These all have to be synchronized with other types of measurements, such as physiological factors (heart rhythm, body-surface humidity and temperature, oxygen consumption, EMG, ECG, EEG, etc.) and environmental factors (temperature and humidity, wind speed, lighting and geometry of the terrain, etc.). Moreover, related qualitative or symbolic information may also be of interest in some cases.

The conventional method for acquiring human motion data is to use dedicated laboratory setups, often including commercial motion capture system, treadmill, and force plates. Such a facility can provide the user with accurate motion reconstruction; however, it is expensive and not always easy to operate. Furthermore, it can only be used for studies under laboratory conditions, whereas there is a rapidly increasing demand for long-term data recording under indoor or outdoor conditions. This implies the need for embedded sensors that are more "wearable," in the sense of delivering maximum autonomy (with long operating times) and transparency (for preserving the natural behavior of the subject). Small, lightweight sensors and batteries are, therefore, essential in order to simplify their integration into shoes [2] or sportswear textiles, for example. Another required quality of such systems is robustness when faced with extreme environmental conditions, such as temperature variations, repeated impacts, dust, sand wind, etc. without excessive deterioration in the data quality.

Inertial measurement units (IMU)-based microsensors are good candidates for meeting such a technological challenge. Indeed, highperformance IMU that combine accelerometers and gyrometers have been widely used, for decades, in the aerospace industry. Microelectromechanical systems-based IMU are small-size devices that are often combined with other kinds of microsensors, such as magnetometers, in order to constitute low-cost-integrated attitude measurement units. There are several providers of such devices worldwide (see, for example, [3]-[5], and [6]). Their performance in terms of accuracy and bandwidth is generally sufficient for a wide range of applications, among them guidance and control of small aerial vehicles, motion capture in virtual reality, human-machine interfaces, and analysis of the motion of living organisms, with a strong emphasis on gait analysis (see, [7], [8], and [9] as examples of the many publications available). One current trend is to arrange the sensors within a specific class of network known as a body area network (BAN) [10].

The application reported here falls into the category of long-term analysis of the performance of an athlete and uses a network of embedded sensors. The runner was, thus, equipped with a set of micro IMUs, force sensitive resistors, and physiological and environmental sensors all integrated into his equipment. The goal was to record all the data possible during six days trail in the desert, and then, later to analyze the runner's behavior and performance throughout the race.
This paper describes various aspects of this unique experiment: technological aspects, results, successes, and failures, as well as the lessons to be learnt. It is organized as follows: the next section describes the wireless sensory system that we have designed; Section III describes the experimental validation performed and finally, Section IV presents our discussion and conclusions.

\section{WIRELESS SENSORY SYSTEM}

\section{A. Nodes}

We have developed a distributed recording architecture based on a wireless sensor network (WSN). The objective is to allow long-term monitoring of information of various kinds (biomechanical, physiological, and environmental) relating to human motion under ecological conditions. The choice of wireless sensors had to facilitate their integration into clothing and provide high connection reliability; wired sensors, for example, could have been expected to break quickly due to mechanical stress. We have also designed a distributed recording architecture, as opposed to a centralized one, simply because the amount of data that would have been needed to be transferred to the collecting point was far too high for the wireless data transfer rate (less than $250 \mathrm{kbits} / \mathrm{s})$. This architecture is based on three kinds of nodes:

1) Motion sensing nodes: each including a three-axis accelerometer, a three-axis gyrometer (composed of two separate chips), and a three-axis magnetometer, the combination of which can be used to estimate the orientation of the unit;

2) Insole pressure sensing nodes: these are composed of six independent force-sensitive sensors (FSR). The six sensors are integrated in the shoe insoles;

3) Master node: this is responsible for network operating and monitoring, environmental measurements (luminosity, temperature, altitude, and humidity), and heart rate monitoring.

These three types of nodes are based on the same core technology, in order to optimize the efficiency of hardware and software development. This core is composed of a 16-bit microcontroller (TI MSP430) and a $2.4 \mathrm{GHz}$ radio transceiver (TI CC2500). It is capable of acquiring data from various kinds of sensors using standard communication buses and interfaces (SPI, I2C, ADC, etc.), of synchronizing with the master node - in order to have consistent timing — and of writing to a micro SD flash card using the FAT32 file system. We have chosen this rather old file system because it is easy to implement on an embedded hardware device with limited capabilities. Furthermore, it has almost no overhead when writing and this file system is readable on most common operating systems.

The main parameters to optimize in the design of the wireless sensor nodes were: size, weight, and power consumption. Since the runner was supposed to be self-sufficient over the six days, the overall weight of the system needed to be as low as possible: this meant that the electronic boards must be small and light, and should not consume excessive energy (to reduce battery size and weight). We have dealt with these constraints and developed a $3.5 \mathrm{~cm} \times 1.5 \mathrm{~cm}$ board that weights $6 \mathrm{~g}$, so that the entire system weights around $150 \mathrm{~g}$ without batteries. A photograph of an IMU node with its micro SD card is shown in Fig. 1. We used batteries with a capacity of $1.2 \mathrm{Ah}$. In order to decrease the consumption of the devices, the time synchronization protocol and the activity scheduling of the sensor nodes were optimized. Regarding the time synchronization protocol, the inter emission period of timestamp packets was computed to minimize the time spent in reception mode by the sensor nodes. In particular, at the network bootstrap and before it gets synchronized, the sensor nodes wait for the reception of a packet coming from the master node to enter the synchronized mode. However, as they cannot remain in reception mode indefinitely waiting 


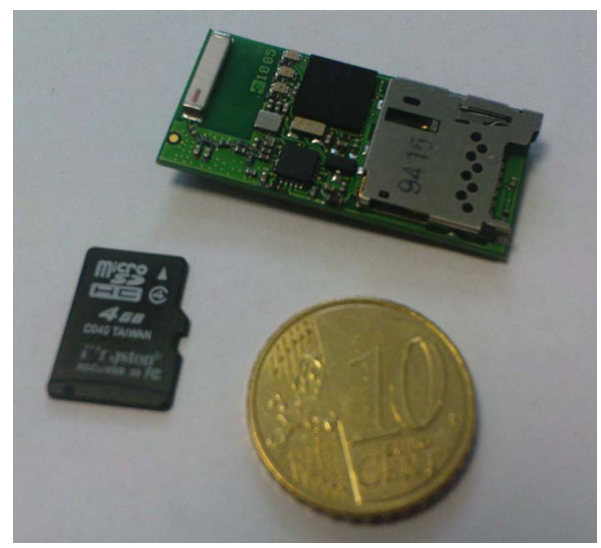

Fig. 1. IMU node with its micro SD card compared to 10 cents of euro coin.

for this reception, we had to setup a rendezvous protocol with sleep periods [11]. Such protocols induce a clear tradeoff between the delay before the rendezvous occurs and the frequency of packet transmission. After implementation, the consumption of the sensor nodes was experimentally measured, giving a $24 \mathrm{~h}$ lifetime with the $1.2 \mathrm{Ah}$ batteries. In consequence, the runner started the race with two batteries per sensor node.

\section{B. $B A N$}

As discussed earlier, long-term recording under outdoor conditions introduces severe stresses on the measurement architecture due to the special operating conditions. For example, for the application reported in this paper, the system was supposed to operate and record data over a six days period during which it was subject to the harsh climatic conditions and strenuous conditions of use associated with a multistage race in the desert. Among other things, this involved contact with sand and dust, high temperatures, high mechanical stresses such as repeated shocks induced by the mechanics of running, partial immersion in sweat, indeed, water whenever the runner used his residual water in a desperate attempt to cool down his body at a check point, etc.

Under such stresses, temporary or permanent failure of a node is not simply a risk but rather a highly probable event that needs to be considered seriously when estimating the system life cycle. In order to reduce the possibility of the entire system being compromised by failure at a single point, we have chosen to design an almost completely distributed system. In such a system, each individual sensor node operates autonomously and independently from all the others. It is well accepted that the distributed paradigm offers much greater reliability and resilience to faults than a centralized paradigm where the integrity of the whole system is reliant on the correct functioning of a single or few elements.

In consequence, the sensor nodes have been designed to operate independently from each other. As described in the previous section, each sensor node possesses its own battery as well as enough storage capacity to fulfill its nonstop task, a closed loop (measure then record) without the need of an external component. Such a simplistic distributed system would have been ideal except in terms of data synchronization.

Let us remember that the objective of the system is to acquire data of various kinds, biomechanical, physiological, and environmental from several physical locations on the runner's body. The power of such a global system requires the capability to analyze these data with a coherent multidimensional approach, e.g., estimating joint angles requires IMU data from each pair of adjacent body limbs. In consequence, if measured independently, all the recorded data must somehow be synchronized so that the offline analysis can be performed over a common global timeline.

Due to the time drift of the internal clocks, the sensor nodes do not share a global clock. Hence, synchronization must be performed by exchanging information, in the form of timestamps, via the wireless channel, the only way in which the sensor nodes can communicate. Time synchronization in WSNs is a classical problem [12], in particular when it is necessary to merge distributed measurements that have been sampled at different frequencies [13], as in our case.

To ensure data synchronization and correct operation of the entire system, the sensor nodes were compiled into a star network with a single node being selected as the master node while the others acted as slave nodes. The master node had the responsibility for operating the network, and to provide all the sensor nodes with timestamps, so that a common time reference was available at all the sensor nodes. The timestamp packets were sent by the master node with a period of $153.4 \mathrm{~ms}$. This period was chosen based on two arguments: first to optimize the tradeoff between delay and energy consumption before synchronization at network bootstrap and second to ensure a low potential drift among sensors between two timestamps. The clock drift of a sensor having been experimentally measured to be inferior to $30 \mathrm{ppm}$, matching the clock specifications, it gives a potential drift of $9.4 \mu \mathrm{s}$ between two sensors if no timestamp packet is lost.

Once synchronized, the network operates as follows.

1) Master: at regular intervals with period $T$, the master node emits a times tamp packet containing its local time $t_{m}$;

2) Slaves: the slave nodes listen to the radio channel with the same periodicity $T$, to receive the timestamp packets and record their reception at local time $t_{s}$ together with the master time contained in the packet $t_{m}$;

3 ) the slave nodes begin their listening period with an offset $\delta$ from the expected reception time to avoid any loss of synchronization that would be caused by a clock drift.

The timestamp and its local reception time $\left(t_{s}, t_{m}\right)$ are recorded at the slave node in a similar format to the other measurements recorded as $\left(t_{s}\right.$, measurement $)$.

Distributed data resynchronization is then performed offline, based on the common timestamps that have been disseminated by the master node to the slave nodes by radio communication. This synchronization is undertaken by making two assumptions:

1) Homogeneous transmission and reception delays: we assume that the delay due to the radio propagation and the system reception latency of a timestamp packet is the same for all slave nodes;

2) Locally constant-rate clock model: we assume that the clock drift of a node remains constant between reception of any two consecutive radio timestamps.

The first assumption is acceptable since the distances between the master node and the slave nodes are similar and negligible compared to the velocity of radio propagation and since the packet reception occurs during an interrupt handler routine that preempts all other tasks in the slave nodes firmware. The second assumption is also realistic because the clock drift variation at a node is mainly due to changes in supply voltage, temperature, etc. Such changes occur at a negligible frequency compared to the times tamp packet period $153.4 \mathrm{~ms}$, and the potential maximum drift of a clock $30 \mathrm{ppm}$. In consequence, we may reasonably expect the clock drift to vary in a negligible way between the reception of two timestamp packets. As an illustration of this offline data synchronization, consider a measurement $m$ performed at local time $t_{s}(m)$. Consider the closest preceding timestamp $t_{m}^{1}$ received at local time $t_{s}\left(t_{m}^{1}\right)$ and the consecutive one $t_{m}^{2}$ received at $t_{s}\left(t_{m}^{2}\right)$. In the 
TABLE I

DESCRIPTION OF THE DIFFERENT SENSOR MODALITIES AND RECORDING CHARACTERISTICS

\begin{tabular}{|c|c|c|}
\hline & Sampling rate (Hz) & Sample size (bits) \\
\hline 3-axis accelerometer & $100 \mathrm{~Hz}$ & 6 \\
\hline 3-axis gyrometer & $100 \mathrm{~Hz}$ & 6 \\
\hline 3-axis magnetometer & $50 \mathrm{~Hz}$ & 6 \\
\hline 6-point insole & $100 \mathrm{~Hz}$ & 12 \\
\hline Temperature & $\frac{1}{16} \mathrm{~Hz}$ & 1 \\
\hline Humidity & $\frac{1}{16} \mathrm{~Hz}$ & 1 \\
\hline Heart rate & $2 \mathrm{~Hz}$ & 1 \\
\hline
\end{tabular}

global reconstructed timeline, the measurement $m$ is located at

$$
t_{G}(m)=t_{m}^{1}+\frac{\left(t_{m}^{2}-t_{m}^{1}\right)}{\left(t_{s}\left(t_{m}^{2}\right)-t_{s}\left(t_{m}^{1}\right)\right)}\left(t_{s}(m)-t_{s}\left(t_{m}^{1}\right)\right) .
$$

Of course, the setup of this data synchronization protocol conflicts with the distributed paradigm that we defended at the beginning of this section. Whereas all sensor nodes are independent in terms of their internal measurement and recording tasks, the data synchronization relies on a centralized protocol and, thus, on a centralized unit, i.e., this is a weak point in the system. Although fully distributed protocols are available, a centralized one was selected despite the reliability risk for several reasons. First, this centralized protocol has the major advantage of simplicity. It reduces the risk of implementation bugs, a not insignificant advantage. Next, it enables a smooth bootstrap for the system. On wakeup, a slave node needs only to wait for reception of its first timestamp packet for it to enter the system and become synchronized. This synchronization period has even been made energy efficient by using a probabilistic listening scheduling [11]. Moreover, it does not require the introduction of a medium access control or collision avoidance protocol; the master node transmits while the slave nodes only receive. Finally, this protocol is energy efficient, since it reduces the volume of radio communication to a minimum.

\section{Different Embedded Sensory Modalities}

As described in Section II-A, the sensor nodes record data from various sources. In order to preserve the spectral properties required by the processing, these data must be acquired with a specific sampling rate. Table I gives the list of sampling rates for each sensor modality and the size of one sample. The sampling rate was chosen according to the modality specificity in terms of type of information and based on our experience and literature. This table gives a precise estimate of the huge amount of data that is collected in $1 \mathrm{~h}$ by one inertial sensor: about 5,5 MB (mega byte). This corresponds to about $175 \mathrm{MB}$ for $32 \mathrm{~h}$ of race.

\section{Packaging}

The purpose of integrating the sensors into the running suit was twofold: to locate all of the nodes efficiently from a measurement point of view, while minimizing the discomfort induced by their inclusion (they must not cause injury due to friction between the sensors and the skin, nor increase the weight of the shoes through addition of sensors and batteries) in order to preserve the natural behavior of the runner. For the master node, the aim was to measure the environmental conditions that the runner was experiencing. Therefore, the board needed to be remote from the runner's body to avoid disturbing these temperature and humidity measurements. The decision was taken to install the

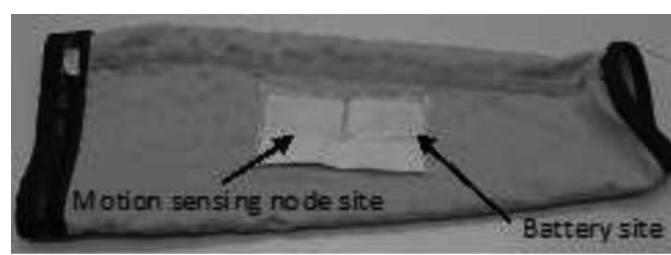

Fig. 2. Motion sensing nodes integration in the race suit.

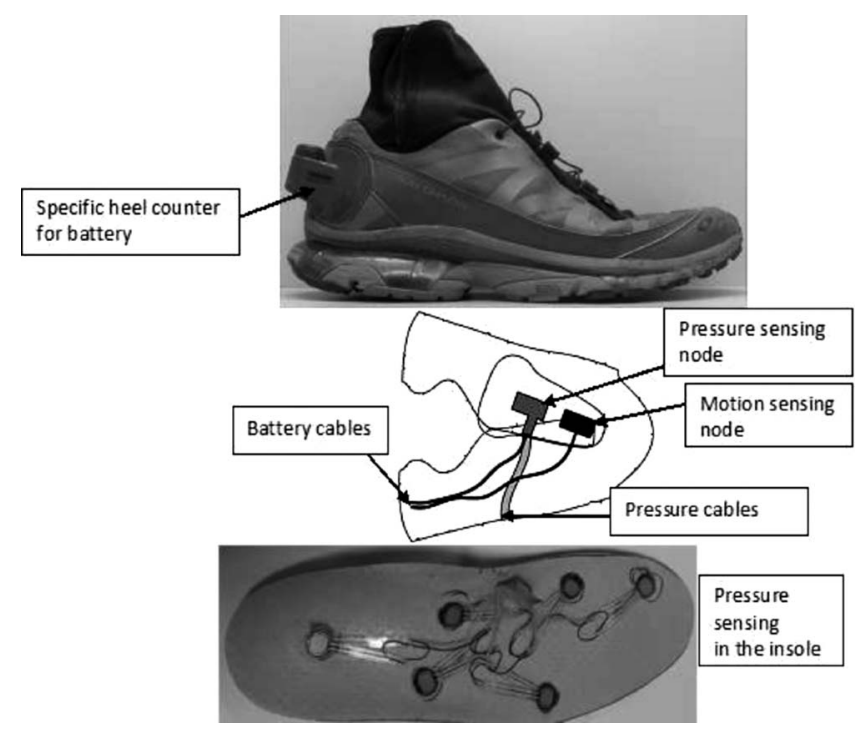

Fig. 3. Inertial and FSR sensors shoe integration.

master node on the backpack shoulder strap. A hole was drilled in the plastic box in order to allow air circulation inside and to obtain a genuine humidity measurement. The temperature measured under these conditions was not a conventional in-the-shade measurement, rather it reflected the actual temperature experienced by the runner. The inertial sensors (motion sensing nodes) were spread across the entire body (except the feet); the objective being to place sensors on the most distal part of each limb possible, while avoiding disrupting movements between the measured limb and the sensor (the sensor was placed on a bony area and not on a muscle). All sensors were placed under a compression garment (EXO Sensifit, Salomon Corporation, Annecy, France) specially designed for this study, in order to secure the sensors on the limbs (see Fig. 2). To minimize the risk of unwanted movement between the limbs and inertial sensors, the battery was placed outside the measurement box and connected to the sensor unit through an extension cable. When incorporating the FSR and inertial sensors into shoes, the aim was to avoid creating discomfort for the runner; therefore, the inertial sensor was installed on the most distal foot area and did not increase the weight of the shoe. In order to meet these requirements, the two FSR and inertial boards were integrated into the shoe tongue (at the most distal point possible) and the batteries were located in an external heel counter, designed for this study (see Fig. 3). All the necessary cables between the inertial sensors, the FSR sensors, and the batteries were inserted into the upper part as illustrated in the figure. Finally, the weight of the shoe (excluding batteries) was the same as that of a shoe from the Salomon XT Wings range designed for this activity (415 $\mathrm{g}$ for a UK size 10 ), and did not modify the overall kinematics of the runner. 


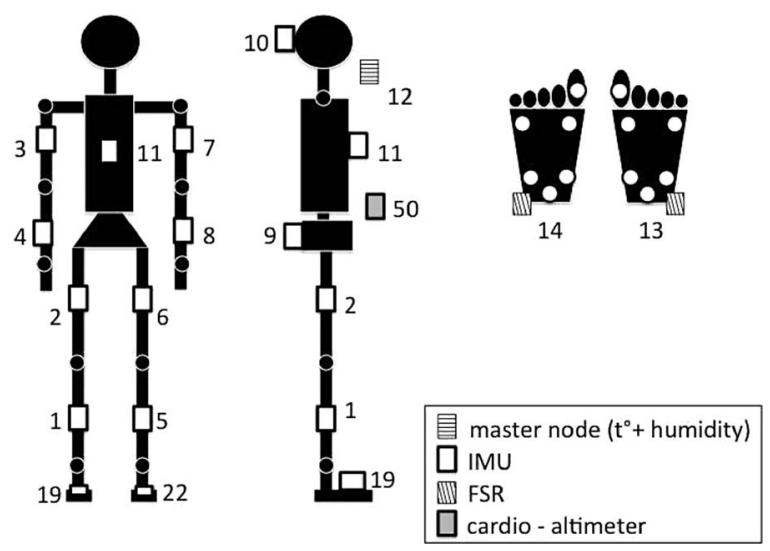

Fig. 4. Nodes disposition on the runner.

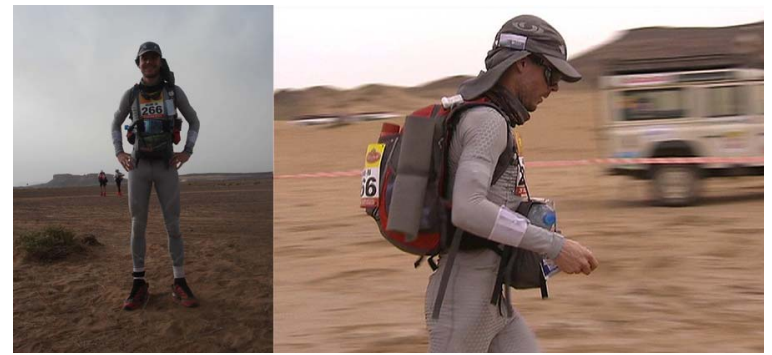

Fig. 5. Runner equipped with the system during the marathon.

\section{EXPERIMENTAL VALIDATION}

\section{A. Experimental Context}

The system was initially tested during several testing campaigns before the race. The testing campaigns took place in several geographic locations and in different conditions (indoor, outdoor, trail, sand, etc.) [14], [15]. The system was then embedded on a runner during the 26th Sultan Marathon Des Sables (MDS). The MDS is a foot race over a distance of about $240 \mathrm{~km}$ in the Moroccan desert. Each participant carries his/her own backpack containing food, sleeping gear, and other material. The participant in the race was authorized by the organizers to use the sensory equipment. His body limbs were entirely instrumented, apart from his hands (see Figs. 4 and 5). The choice of this race is justified by the objective of the experiment: to show that such sensory system can be used in real conditions and over a long period without specific human intervention or maintenance. And this race was perfect for that it lasts six days, it is run in total autonomy and in very hard conditions, and the temperature raising up to $50{ }^{\circ} \mathrm{C}$. It induced strong constraints on the system: it has to be energy efficient, it should be ergonomic and comfortable enough not to disturb or hurt the runner, and it has to be robust enough to survive the shocks, the temperature, the sweet, and the manipulation (when dressing and undressing).

\section{B. Results}

Our runner finished the competition ranked 66 out of 1013 competitors, 923 of whom completed all six stages. The feedback from the runner was very positive: the system was comfortable to wear and the sensors were small and light enough so that they did not disturb him. The only default of the device was a wire that was misplaced in one of the pressure insoles that caused damages to the runner feet.
TABLE II

RACE STAGES DESCRIPTION AND CORRESPONDING NODES WHICH CORRECTLY ACQUIRED DATA

\begin{tabular}{|l|c|c|c|}
\hline Stage & Distance & Duration & Functional nodes (node success ratio) \\
\hline 1 & $29 \mathrm{~km}$ & $3 \mathrm{~h} 30$ & $1,3,8,9,10,12,19,14^{*}, 15(56 \%)$ \\
\hline 2 & $35 \mathrm{~km}$ & $4 \mathrm{~h} 54$ & $1,3,9,10,12(31 \%)$ \\
\hline 3 & $40 \mathrm{~km}$ & $5 \mathrm{~h} 08$ & $12(6 \%)$ \\
\hline 4 & $82 \mathrm{~km}$ & $11 \mathrm{~h} 58$ & $7,12,19^{*}(19 \%)$ \\
\hline 5 & $42 \mathrm{~km}$ & $4 \mathrm{~h} 43$ & $3,7,12,19(19 \%)$ \\
\hline 6 & $22 \mathrm{~km}$ & $2 \mathrm{~h}$ & $12(6 \%)$ \\
\hline
\end{tabular}
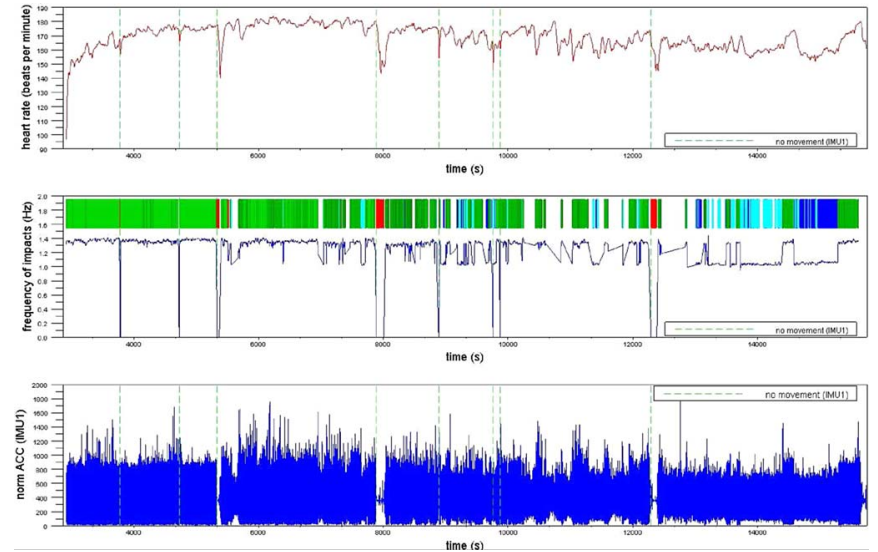

Fig. 6. Example of post-collection analysis of data recorded during the stage 1 of the competition. (Top) Heart rate recorded by node 12. (Middle) Frequency of the impacts detected by FSR sensors (autocorrelation of the signals). Segmentation result at the top part of the graph: green stands for running classes, blue for walking classes, and red for breaks (runner is not moving). (Bottom) Norm of the three accelerometers of node 1 .

1) Recorded Data: the distances and durations of all stages are reported in Table II, together with the references of the nodes for which data were correctly collected. A connection problem between measurement units and batteries was responsible for difficulties with the power supply to each individual node, except for node 12 which was not using any extension cable and worked perfectly throughout the entire race. Therefore, during some parts of the various stages, full sets of data were recorded. Furthermore, had the power supply been disrupted during the SD card writing, the whole file could have been corrupted. This packaging weakness was responsible for the high failure rate that was observed over the whole competition.

2) Examples of Estimated Parameters: Fig. 6 presents some of the data obtained during stage 1 and their analysis. The computation of the step frequency (middle) is based on an autocorrelation of the signal from the FSR. Even at first glance, this figure shows good coherence between the three types of data (cardiac frequency, feet impacts, and accelerations), with major peaks corresponding to the times when the runner stopped (with the classical delay in the slowing down of the heart rate), and an overall decrease in acceleration toward the end of the race corresponding to a predominance of walking over running. The combination of all these parameters gives a good idea of the state of the runner, with some redundancy useful in the case of sensor failure. Such data can also be used to separate walking and running phases, and the different velocities. Usually, discrimination between walking and running can be performed by measuring the double support duration. However, since the finally available FSR data came from a single shoe only, we had to use a segmentation approach based on the step period only, which is known to be greater when walking than when running, combined in some cases with the acceleration. If a 

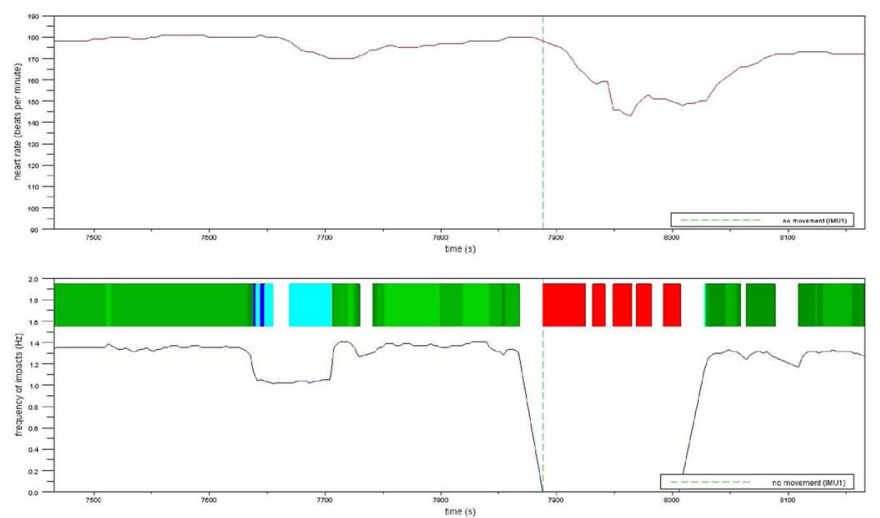

Fig. 7. Zoom on Fig. 6. (Top) Heart rate recorded by node 12. (Bottom) Frequency of the impacts detected by FSR sensors (autocorrelation of the signals). Segmentation result at the top part of the graph: green stands for running classes, blue for walking classes, and red for breaks.

more accurate labeling of the running phases is desired, into different velocity classes, then step length is relevant rather than step period. In that case, a segmentation method based on the detection of abrupt changes to the norm of the acceleration of the right shank could be used. Fig. 6 also illustrates the results for the case of the three simplest classes: running, walking, and stopped. The magnified section in Fig. 7 shows the following sequence: running, walking, running, stopped, and running again. Blank periods indicate when no classification could be achieved. For more details and other results on segmentation methods, the reader is referred to [14].

\section{DISCUSSION AND CONCLUSION}

We have developed a new BAN based on wireless sensors. The network has been optimized in order to allow for long-term recording under extreme conditions. Other similar products such as Shimmer Research or XSens are available in the market. From an hardware point of view, our IMUs are similar but we have extended the principle to other sensor types such as feet pressure and environmental parameters. Our device also greatly differs from these products since it operates as a whole sensor network, providing synchronized data from all over the runner's body, and not as single independent devices. The integration of the sensors in the racer equipment, especially in the insoles and running shoes, is also an originality of our system. The optimization of the embedded software, the communication and time-synchronization protocols, and the low energy consumption of the devices for the sixstage race are also specific to our system. There is a big difference between a system that can be used $3 \mathrm{~h}$ in a laboratory and one that can be deployed for a long period in hard conditions and without human intervention. Our objective was to walk this step. From a runner point of view the system is a success, it is comfortable and light.

The encountered power loss problems can be ameliorated through the design of a better packaging. An extension cable with a connector was used between the sensors and batteries. This connector was the weak link of the system regarding robustness. Indeed, the connector was neither protected by a hard box, as the sensor was, nor sunk into silicon, as the battery was. It was only protected by the sensor pockets in the race suit. In consequence, the connector was neither waterproof nor shock proof and we suffered disconnections and, hence, loss of power. There is no easy solution to prevent files from getting corrupted upon a power loss. Indeed, if the power loss occurs between two SD card writings, there is no corruption: the file system had been made robust so that the files are not lost when the sensor is violently rebooted.
However, when the power loss occurs during a write access, the data are lost and the file gets corrupted.

The types of sensors that could be incorporated in such a network are not limited to those described here. Indeed, most of the sensors that are conventionally used in human movement science could be plugged into the generic amplifier nodes (GPS, goniometer, EMG, etc.). From the user's point of view, a second goal of the experiment was to validate the processing algorithms dedicated to the automatic analysis of large amounts of data collected under extreme conditions. For illustration, in this paper we have presented some examples of basic data analysis, which could obviously be extended according to the target applications. The system is currently being used in several other studies in the domains of animal biologging and functional rehabilitation. As an example, it equips cats in the context of a study on their preying habits conducted by the CERFE. The experiment presented in this paper will also be renewed during the Ultra Trail du Mont Blanc, a $160 \mathrm{~km}$ race around the highest European summit. In the domain of functional rehabilitation, this equipment is now used by the INRIA in order to control assistive devices (electrical stimulation) during sit to stand and walking tasks. Finally, this system is at the center of a technological transfer that has led to the creation of the HIKOB SAS company.

\section{ACKNOWLEDGMENT}

A particular acknowledgment to Darbaroud, the 25th Sultan MDS organization committee, for their support and for having accepted such a strange runner in the race.

\section{REFERENCES}

[1] D. Gouwanda and S. M. N. A. Senayake, "Emerging trends of bodymounted sensors in sports and human gait analysis," in Proc. Int. Conf. Biomed. Eng., Kuala Lumpur, Malaysia, 2008, pp. 715-718.

[2] S. Bamberg, A. Benbasat, D. Scarborough, D. Krebs, and J. Paradiso, "Gait analysis using a shoe-integrated wireless sensor system," IEEE Trans. Inf. Technol. Biomed., vol. 12, no. 4, pp. 413-423, Jul. 2008.

[3] XSENS Products, (2011). [Online]. Available: http://www.xsens.com

[4] MOVEA Company, (2011). [Online]. Available: http://www.movea.com

[5] MICROSTRAIN Company, (2011). [Online]. Available: http://www. microstrain.com

[6] SBG systems, (2011). [Online]. Available: http://www.sbg-systems.com

[7] R. Héliot, C. Azevedo-Coste, L. Schwirtlich, and B. Espiau, "Gait spectral index (GSI): A new quantification method for assessing human gait," Health, vol. 2, no. 1, pp. 38-44, 2010.

[8] W. Zijlstra and A. L. Hof, "Assessment of spatio-temporal gait parameters from trunk accelerations during human walking," Gait Posture, vol. 18, pp. 1-10, 2003.

[9] M. Brandes, W. Ziljstra, S. Heikens, R. van Lummel, and D. Rosenbaum, "Accelerometry-based assessment of gait parameters in children," Gait Posture, vol. 24, pp. 482-486, 2006.

[10] S. Hu and J. Tan, "A wireless physiological sensing and logging system with applications in poultry science," in Proc. IEEE Eng. Med. Biol. Soc. Conf., 2009, pp. 4828-4831.

[11] E. Ben Hamida, G. Chelius, A. Busson, and E. Fleury, "Neighbor discovery in multi-hop wireless networks: Evaluation and dimensioning with interferences considerations," Discr. Math. Theor. Comput. Sci., vol. 2, pp. $87-114,2008$.

[12] K. Römer, P. Blum, and L. Meier, "Time synchronization and calibration in wireless sensor networks," in Handbook of Sensor Networks: Algorithms and Architectures, I. Stojmenovic, Ed. New York: Wiley, Sep. 2005, pp. 199-237.

[13] D. Ganesan, S. Ratnasamy, H. Wang, and D. Estrin, "Coping with irregular spatio-temporal sampling in sensor networks," ACM SIGCOMM Comput. Commun. Rev., vol. 34, no. 1, pp. 125-130, 2004.

[14] M. Pasquier, B. Espiau, and C. Azevedo-Coste, "Signal-based segmentation of human locomotion using embedded sensor network," in Proc. IEEE Int. Conf. Acoust., Speech, Signal Process., Prague, Czech Republic, May 2011, pp. 669-672.

[15] XTREMLOG project, (2010). [Online]. Available: http://www.youtube com/user/xtremlog 